\title{
State-Salient Decision Rules for Choice under Uncertainty
}

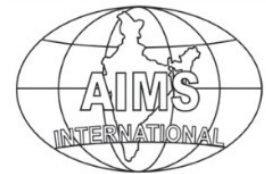

DOI: $10.26573 / 2019.13 .3 .3$

Volume 13, Number 3

September 2019, pp. 191-195

\author{
Somdeb Lahiri \\ SPM, PDP University \\ Gandhinagar, India \\ (somdeb.lahiri@gmail.com)
}

We suggest that the celebrated Impossibility Theorem of Arrowian social choice theory is after all not such a negative result since it can be interpreted as an axiomatic characterization of state-salient decision rules of a decision maker with state-dependent rankings of alternatives. We provide examples to show that there are meaningful choice functions which are not state-salient decision rules.

Keywords: Choice Function, Arrow's Impossibility Theorem, State-Salient Decision Rules

\section{Introduction}

One implication of Arrow's Impossibility Theorem for which an eclectic and reasonably up-to-date bibliography of the vast literature associated with it is available at https://plato.stanford.edu/entries/arrows-theorem/, may be that the underlying framework is not appropriate for the study of group decision making. Perhaps group decision making usually does not take place that way. I don't know why I have never come across such a statement. Ignorance on my part about comments concerning the Impossibility Theorem and the fact that I may not have been able to appreciate the underlying philosophy (not the proof) of the said theorem are equally likely reasons for me to have never seen a statement about the inappropriateness of the Arrowian framework for the study of group decision making. In any case, I don't think there is any harm in suggesting an alternative interpretation that works.

This paper is just that- an interpretation of the Arrowian framework and the impossibility theorem that (also?) makes good sense. We provide a realistic example that satisfies the conditions of the theorem and to emphasize that the theorem is meaningful and has content, we provide two examples that do not satisfy the conditions. Let me emphasize that this paper is not intended to be a contribution to the vast literature on social choice theory, and its purpose would be defeated if one tried to view it that way. It is a paper concerned with decision making under risk and uncertainty.

\section{The Framework and the Main Result}

The motivation for the framework of this note can be found in a recent work (Lahiri 2019). 
Let $\mathrm{X}$ be a non-empty finite set of alternatives containing at least three elements, from which a decision maker is required to choose exactly one alternative. There is a non-empty finite set of future states of nature, which unravel only after the decision maker has made his/her choice. For a positive integer $n \geq 3$, let $N=\{1,2, n\}$ denote the set of states of nature.

A strict (preference) ranking on $\mathrm{X}$ is a reflexive, complete/connected/total, transitive and anti-symmetric binary relation on $\mathrm{X}$. A strict ranking is also known as a linear order. Generally a preference relation is denoted by $R$ with $P$ and $I$ denoting its asymmetric and symmetric parts respectively. If for $x, y \in \mathrm{X}$, it is the case that $(x, y) \in R$, then we shall denote it by $x R y$ and say that $x$ is at least as good as $y$. Similarly $x P y$ is interpreted as $x$ is strictly preferred to $y$, and $x I y$ is interpreted as there is indifference between $x$ and $y$.

Let $\mathcal{L}$ denote the set of all strict rankings on $\mathrm{X}$.

A (state-dependent) preference profile denoted $R_{N}$ is a function from $\mathrm{N}$ to $\mathcal{L}$. $\mathrm{R}_{\mathrm{N}}$ is represented as the array $\left\langle R_{i} \mid i \in \mathrm{N}\right\rangle$, where $R_{i}$ is the strict ranking of the decision maker of the alternatives in $\mathrm{X}$ in state of nature $i$. The set of all preference profiles is denoted $\mathcal{L}^{\mathrm{N}}$.

A choice function $(\mathrm{CF})$ is a function from $\mathcal{L}^{\mathrm{N}}$ to $\mathrm{X}$.

A CF $\mathrm{f}$ is said to satisfy the Weak Dominance Criterion (WDC) if for all $x, y \in \mathrm{X}$ and $R_{N} \in \mathcal{L}^{\mathrm{N}}:\left[x \mathrm{P}_{\mathrm{i}} y\right.$ for all $\left.i \in \mathrm{N}\right]$ implies $\left[\mathrm{y} \neq \mathrm{f}\left(R_{N}\right)\right]$.

This criterion is available and discussed in the aforementioned work (Lahiri 2019). The following criterion is one based on a more general property (Denicolo 1993).

A CF $\mathrm{f}$ is said to satisfy Independence of Irrelevant Alternatives in the sense of Denicolo (D-IIA) if for all $R_{N}, R_{N}^{\prime} \in \mathcal{L}^{\mathrm{N}}$ and $x, y \in \mathrm{X}$ with $x \neq y:\left[R_{i}\left|\{x, y\}=R_{i}^{\prime}\right|\{x, y\}\right.$ for all $\left.i \in \mathrm{N}, x=\mathrm{f}\left(R_{N}\right)\right]$ implies $\left[y \neq \mathrm{f}\left(R_{N}^{\prime}\right)\right]$.

The following definition can be found in the work that has been referred to in this paper twice earlier (Lahiri 2019).

A CF $\mathrm{f}$ is said to be state-salient between a given pair of distinct alternatives at state of nature $i$, if regardless of the state-dependent preference profile, the alternative in the given pair that is ranked inferior of the two in state of nature $i$, is not chosen. Thus, if the two alternatives are $x$ and $y$ and if it is the case that (a) if $x$ is ranked above $y$ in state $i$, then $y$ is not chosen regardless of the rankings in other states of nature, and (b) if $y$ is ranked above $x$ in state $i$, then $x$ is not chosen regardless of the rankings in other states of nature.

A CF $\mathrm{f}$ is said to be state-salient at a state of nature $i$, if it is state salient between every pair of distinct alternatives at state of nature $i$.

A CF $\mathrm{f}$ is said to be a state-salient decision rule (S-SDR), if there exists a state of nature $i$, such that $\mathrm{f}$ is state salient at state of nature $i$.

An important and realistic example of an S-SDR is the maximum likelihood decision rule.

A CF $\mathrm{f}$ is said to be a maximum likelihood state decision rule (MLSDR) if there exists a probability distribution $\mathrm{p}$ on $\mathrm{N}$ (i.e. $\mathrm{p} \in \mathbb{R}_{+}^{\mathrm{N}}$ with $\sum_{i=1}^{\mathrm{n}} \mathrm{p}_{\mathrm{i}}=1$ ) and a state of nature $i$ such that $\mathrm{p}_{i} \geq \mathrm{p}_{j}$ for all $j \in \mathrm{N} \backslash\{i\}$ (i.e. $i \in \underset{j \in \mathrm{N}}{\operatorname{argmax}}\left\{\mathrm{p}_{j}\right\}$ ) such that for all $R_{N} \in \mathcal{L}^{\mathrm{N}}$ and $x, y \in \mathrm{X}$ : $x \mathrm{P}_{i} y$ implies $y \neq \mathrm{f}\left(R_{N}\right)$. In this case $i$ is said to be a most likely state of nature (MLS). 
The version of MLSDR when state-dependent rankings are represented by statedependent evaluation or utility functions is available on page 74 in chapter 4 of a book concerned with quantitative methods in decision making (Mc Kenna 1980) under the name "maximum likelihood criterion". The same is also defined and discussed under a different name - "modal outcome criterion"- on page 429 (section 9.4.2) of a book on managerial decision making (Monahan 2000). However as correctly observed by several authors, using this criterion in a situation where several states of nature exist, with probability of occurrence nearly or exactly equal to the probability of occurrence of a most likely state of nature, may lead to serious mistakes. The following example illustrates the plausibility of an MLSDR as a decision aid/decision support method under appropriate conditions.

Example 1: Consider a typical decision making problem that is faced by many residents living in the southern Indian coastal state of Kerala, before leaving home for work in the morning. The weather in Kerala from May 1 to June 30 passes through three distinct phases. The first three weeks of May are hot and sunny and there is usually no rain during this period. During the last week of May and the first week of June the weather is quite unpredictable with near equal likelihood of the day being sunny or rainy. Thereafter, the monsoon that Kerala is famous for sets in with full gusto.

Before leaving for work several individuals in Kerala are often faced with the problem of choosing exactly one alternative from the set $\mathrm{X}=$ \{raincoat, umbrella, hat\}, in order to protect themselves from the intense vagaries of nature which depends on whether the day ahead is going to be rainy or sunny. Although, few would like to be drenched in the rain with a hat on his head or risk getting a sunstroke by wearing a raincoat in the scorching sun, we shall allow for all possible state-dependent individual preferences. Thus in each state of nature, (i.e. rainy or sunny), there are a six possible rankings of the three alternatives in the set X. During the first three weeks of May, most individuals will proceed to work under the assumption that they have to face scorching sun while travelling and will thus carry with them what they prefer most on sunny days- usually either an umbrella or a hat. Similarly during the last two or three weeks of June, most individuals will set out for work assuming they will have to play hide-and-seek with rain on the way, and would thus equip themselves with what they prefer most on a rainy day- usually a rain-coat or an umbrella. However, since the weather is unpredictable during the last week of May and the first week of June, deciding on the most appropriate mode of protection from extreme weather conditions is really a guessing game, and one may face unexpected consequences if one decides on a most preferred alternative for a state of nature which has a $51 \%$ chance of being realised during the day.

The following is the main result based on a theorem due to Denicolo (1993).

Theorem 1: A CF satisfies WDC and D-IIA if and only if it is any S-SDR.

\section{Choice Functions which are not S-SDR's}

As for all other meaningful solution concepts, there are many realistic choice functions which do not fall in the category of an S-SDR. The following leads to such a choice function. 
Given any strict ranking $R$ on $\mathrm{X}$, let first $(R)$ denote the alternative that is ranked first (at the top) by $R$, i.e. $x P y$ for all $y \in \mathrm{X} \backslash\{x\}$.

Let $\mathrm{p}$ be any probability distribution on $\mathrm{N}$ and suppose that $i$ is a state of nature such that $\mathrm{p}_{i} \geq \mathrm{p}_{j}$ for all $j \in \mathrm{N} \backslash\{i\}$, i.e. $i$ is a most likely state of nature for $\mathrm{p}$.

Let $\widehat{\mathrm{fp}^{\mathrm{p}}}$ be the CF such that for all $R_{N} \in \mathcal{L}^{\mathrm{N}}, \widehat{\mathrm{fp}}\left(R_{N}\right)=x$ if and only if either $\sum_{j \in\left\{k \in \mathrm{N} \mid \text { first }\left(R_{k}\right)=x\right\}} \mathrm{p}_{j}>\frac{1}{2}$ (i.e. the probability that the state of nature will be such that $x$ is the best choice is greater than $\frac{1}{2}$, in which case $x$ will of necessity be the unique alternative to satisfy this property) or $\left\{y \in \mathrm{XI} \sum_{j \in\left\{k \in \mathrm{N} \mid \mathrm{first}\left(R_{k}\right)=y\right\}} \mathrm{p}_{\mathrm{j}}>\frac{1}{2}\right\}=\phi$ and first $\left(R_{i}\right)=x$.

Clearly, $\widehat{\mathrm{f}^{\mathrm{p}}}$ satisfies WDC. However it does not satisfy D-IIA and is thus not a S-SDR as the following example reveals.

Example 2: Let $\mathrm{X}=\{x, y, z\}$ and $\mathrm{N}=\{1,2,3\}$. Let $\mathrm{p}_{1}=\frac{2}{5}, \mathrm{p}_{2}=\mathrm{p}_{2}=\frac{3}{10}$. Clearly 1 is the most likely state of nature for $\mathrm{p}$. Suppose $x R_{1} y R_{1} z, z R_{2} x R_{2} y, y R_{3} z R_{3} x$. Since none of the alternatives is ranked first with probability greater than half, $\widehat{\mathrm{f}^{\mathrm{p}}}$ chooses $\mathrm{x}$ and $\mathrm{z}$ is not chosen.

Now suppose we have a preference profile where in the first and second states of nature, the rankings are identical to the ones in the earlier situation. However in the third state of nature $y$ and $z$ interchange positions. In this new profile the probability that $z$ will be ranked first is $\frac{3}{5}$ which is greater than half. Hence $\widehat{\mathrm{f}^{\mathrm{p}}}$ chooses $z$ now, although in both the profiles the relative positions of $x$ and $z$ are the same.

Thus, $\widehat{\mathrm{f}}$ violates D-IIA and by Theorem 1, it cannot be a state-salient decision rule.

A CF $\mathrm{f}$ is said to satisfy the maximum likelihood alternative property (MLA) if there exists a probability distribution $\mathrm{p}$ on $\mathrm{N}$, such that for all $R_{N} \in \mathcal{L}^{\mathrm{N}}, \mathrm{f}\left(\mathrm{R}_{\mathrm{N}}\right)=x$ implies $x \in \underset{y \in \mathrm{X}}{\operatorname{argmax}} \sum_{j \in\left\{k \in \mathrm{N} \mid \mathrm{first}\left(R_{k}\right)=y\right\}} \mathrm{p}_{j}$.

A CF that satisfies MLAP is the following.

Given a probability distribution $\mathrm{p}$ on $\mathrm{N}$, the $\mathrm{CF} \overline{\mathrm{f}}^{\mathrm{p}}$ is such that for all $R_{N} \in \mathcal{L}^{\mathrm{N}}$, $\overline{\mathrm{f}} \mathrm{p}\left(R_{N}\right)=x$ if and only if:

i. $\quad x \in \underset{y \in \mathrm{X}}{\operatorname{argmax}} \sum_{j \in\left\{k \in \mathrm{N} \mid \operatorname{first}\left(R_{k}\right)=y\right\}} \mathrm{p}_{j}$

ii. $\quad \operatorname{Min}\left\{j \in \mathrm{N} \mid\right.$ first $\left.\left(R_{j}\right)=\mathrm{x}\right\} \leq \min \left\{j \in \mathrm{N}\right.$ lfirst $\left.\left(R_{j}\right)=\mathrm{z}\right\}$ for all

$z \in \underset{y \in \mathrm{X}}{\operatorname{argmax}} \sum_{j \in\left\{k \in \mathrm{N} \mid \operatorname{first}\left(R_{k}\right)=y\right\}} \mathrm{p}_{\mathrm{j}}$.

If $\left.\underset{y \in \mathrm{X}}{\operatorname{argmax}} \sum_{j \in\{k \in \mathrm{N} \mid \operatorname{first}}\left(R_{k}\right)=y\right\}$ 的 is a singleton then (ii) becomes redundant.

Clearly, $\overline{\mathrm{f}}^{\mathrm{p}}$ satisfies WDC. However it does not satisfy D-IIA and is thus not a

S-SDR as the following example reveals.

Example 3: Consider the scenario in example 2, i.e. $\mathrm{X}=\{x, y, z\}, \mathrm{N}=\{1,2,3\}, \mathrm{p}_{1}=\frac{2}{5}$, $\mathrm{p}_{2}=\mathrm{p}_{2}=\frac{3}{10}$. Suppose $x R_{1} y R_{I} z, z R_{2} x R_{2} y, y R_{3} z R_{3} x$. Then, $\overline{\mathrm{f}}^{\mathrm{p}}$ chooses $x$ and $z$ is not chosen. 
Now as in example 2, suppose we have a preference profile where in the first and second states of nature, the rankings are identical to the ones in the earlier situation. However in the third state of nature $y$ and $z$ interchange positions. In this new profile the probability that $\mathrm{z}$ will be ranked first is $\frac{3}{5}$, which exceeds the probability that $x$ will be ranked first and also the probability that y will be ranked first. Hence $\overline{\mathrm{f}}^{\mathrm{p}}$ chooses $z$ although in both the profiles the relative positions of $x$ and $z$ are the same. Thus, $\overline{\mathrm{f}}^{\mathrm{p}}$ violates D-IIA and by Theorem 1 , it cannot be a state-salient decision rule.

\section{Acknowledgment}

I would like to thank Professor Prasanta Pattanaik for motivation and inputs (both direct and indirect) in writing this paper. His role as my mentor for decision theory in the Arrow-Sen tradition is undeniable. I would like to thank Carlos Bouza and Roberto Veneziani for much encouragement and encouraging comments while and after writing this paper. I would also like to thank the Editor-in-Chief of this journal, Dr. Omprakash Gupta, for suggesting a new title for this paper, less cryptic than what the earlier one was and for useful comments which have gone a long way in considerably improving the quality of the paper. However responsibility for errors and views expressed in this paper rests entirely on the author of this paper and no one else.

\section{References}

1. Denicolo, V., 'Fixed Agenda Social Choice Theory: Correspondence and Impossibility Theorems for Social Choice Correspondences and Social Decision Functions', Journal of Economic Theory, vol. 59(2), 1993, 324-332.

2. Lahiri, S., 'Re-interpreting the Arrow-Sen framework of social choice' npublished, 2019. https://www.academia.edu/40224623/Reinterpreting_the_Arrow-Sen_Framework_of_Social_Choice

3. Monahan, G., 'Management Decision Making: Spreadsheet Modeling, Analysis, and Application', Cambridge University Press, 2000.

4. Mc Kenna, C., 'Quantitative Methods for Public Decision Making' (First Edition). Mc Graw Hill, NY, 1980.

\section{About Our Author}

Somdeb Lahiri (Ph.D., University of Minnesota, 1986) is a Professor of Economics at the School of Petroleum Management, PDP University (PDPU). His current research interests are in microeconomic theory, with special focus on decision theory. His current teaching portfolio includes microeconomics, mathematical (methods for) economics and management and operations research. 